УДК 621.372 .6

\title{
ШЕСТИВХОДОВОЕ УСТРОЙСТВО НА ОСНОВЕ МНОГОСЛОЙНЫХ ИНТЕГРИРОВАННЫХ В ПОДЛОЖКУ ВОЛНОВОДОВ
}

\author{
Т. Х. БОУАЗ3А ${ }^{1}$, К. НОУРИ, ДЖ. ТАО ${ }^{2}$, Б. С. БОУАЗ3А ${ }^{1}$ И Т.-Х. ВУОНГ \\ ${ }^{1}$ Университет Тахара Моулая, \\ Алжси, Саида, 20000 \\ ${ }^{2}$ ENSEEIHT, \\ Франици, Тулуза, 31071
}

\begin{abstract}
Аннотация. В статье представлена новая конструкция шестивходового устройства на основе многослойных интегрированных в подложку волноводов SIW (substrate integrated waveguides). Эта конструкция основана на использовании многослойной структуры с целью уменьшения размеров устройства при сохранении рабочих характеристик реализуемого компонента. Разработанное шестивходовое устройство на базе SIW состоит из двух базовых элементов: делителя мощности SIW и направленного ответвителя. Эти два элемента спроектированы, оптимизированы и согласованы для получения улучшенных рабочих характеристик на рабочей частоте 11 ГГц. Результаты моделирования показали, что новое шестивходовое устройство на базе многослойного SIW обладает хорошими рабочими характеристиками, в частности хорошим уровнем обратных потерь, развязкой более -20 дБ, и величиной коэффициента передачи выше -10 дБ. Преимуществом этого SIW устройства являются малые размеры $160 \times 34,8$ мм, ширина данного устройства примерно на 50\% меньше, чем у планарного шестивходового SIW устройства, что позволяет достичь более высокой плотности интеграции в телекоммуникационных системах и планировать создание устройств с меньшими размерами. Переход от микрополосковой линии к SIW используется для облегчения интеграции этого компонента в другие планарные устройства. Указанные структуры спроектированы, промоделированы и оптимизированы с помощью программного обеспечения Ansoft HFSS.
\end{abstract}

Ключевые слова: шестивходовый SIW; интегрированный в подложку волновод; многослойный; ответвитель; делитель мощности

\section{1. ВВЕДЕНИЕ}

В последнее время исследовались различные многовходовые компоненты [1-5], которые могут представлять интерес для разработчиков и стремятся удовлетворить постоянно растущие ожидания, возлагаемые на СВЧ устройства.

Шестивходовое устройство представляет собой пассивную линейную СВЧ цепь. Ее разработка продолжалась в течение последних 40 лет для измерительных приложений СВЧ диапазона и диапазона миллиметровых волн [6].

DOI: $10.20535 / \mathrm{S} 0021347018020036$

(C Т. Х. Боуазза, К. Ноури, Дж. Тао, Б. С. Боуазза и Т.-Х. Вуонг, 2018
Многочисленные архитектуры шестивходовых радиоустройств были предложены для разработки дешевых и высокоэффективных трансиверов с прямым преобразованием и характеристиками, определяемыми программным обеспечением (ПО), которые привлекательны для пользователей, поскольку обеспечивают хорошие рабочие характеристики, связанные с потребляемой мощностью, линейностью и шумом системы, а также расходами на проектирование и изготовление.

Шестивходовое устройство может быть построено в виде гибридных частей либо в 


\section{БИБЛИОГРАФИЧЕСКИЙ СПИСОК}

1. Kobrin, K. V.; Manuilov, M. B.; Sinyavsky, G. P. "Field-theory analysis and design of multiport branch-guide couplers for contoured beam antennas," Proc. of Int. Conf. on Antenna Theory and Tech., ICATT, 9-12 Sept. 2003, Sevastopol, Ukraine. IEEE, 2003, pp. 769-771, 2003. DOI: 10.1109/ICATT.2003.1238861.

2. Kantartzis, N. V.; Gatzianas, M.; Kosmanis, T. I.; Tsiboukis, T. D. "Analysis of multiport waveguide structures by a higher-order FDTD methodology based on non-orthogonal curvilinear grids," IEEE MTT-S Int. Microwave Symp. Dig., 20-24 May 2001, Phoenix, AZ, USA. IEEE, 2001, pp. 2051-2054. DOI: 10.1109/MW SYM.2001.967315.

3. Wessel, W.; Sieverding, T.; Arndt, F. "Mode-matching analysis of general waveguide multiport junctions," IEEE MTT-S Int. Microwave Symp. Dig., 13-19 Jun. 1999, Anaheim, CA, USA. IEEE, 1999, pp. 1273-1276. DOI: 10.1109/MWSYM.1999.779619.

4. Bialkowski, M. E. "Analysis of an N-port consisting of a radial cavity and E-plane coupled rectangular waveguides," IEEE Trans. Microwave Theory Tech., Vol. 40, No. 9, pp. 1840-1843, Sep. 1992. DOI: $\underline{10.1109 / 22.15}$ $\underline{6613}$.

5. Yeo, S. P.; Qiao, L.; Cheng, M. "Symmetrical $\mathrm{N}$-port waveguide junction loaded with dielectric sleeve and metallic post," IEEE Trans. Microwave Theory Tech.,
Vol. 43, No. 6, pp. 1298-1302, Jun. 1995. DOI: 10.1109/ 22.390186 .

6. Engen, G. F. "The six-port reflectometer: An alternative network analyzer," IEEE Trans. Microwave Theory Tech., Vol. 25, No. 12, pp. 1075-1080, Dec. 1977. DOI: 10.1109/TMTT.1977.1129277.

7. Urbanec, T. "Special methods for microwave vector measurements," Ph.D. Thesis, Dept. of Radio Electronics, Brno University of Technology, Brno, Czech Republic, 2007.

8. Deslandes, D.; Wu, K. "Integrated microstrip and rectangular waveguide in planar form," IEEE Microwave Wireless Components Lett., Vol. 11, No. 2, pp. 68-70, Feb. 2001. DOI: $10.1109 / 7260.914305$.

9. Uchimura, H.; Takenoshita, T.; Fujii, M. "Development of a 'laminated waveguide'," IEEE Trans. Microwave Theory Tech., Vol. 46, No. 12, pp. 2438-2443, Dec. 1998. DOI: $10.1109 / 22.739232$.

10. Hirokawa, J.; Ando, M. "Single-layer feed waveguide consisting of posts for plane TEM wave excitation in parallel plates," IEEE Trans. Antennas Propag., Vol. 46, No. 5, pp. 625-630, May 1998. DOI: $10.1109 / 8.668903$.

11. Grigoropoulos, N.; Sanz-Izquierdo, B.; Young, P. R. "Substrate integrated folded waveguides (SIFW) and filters," IEEE Microwave Wireless Compon. Lett., Vol. 15, No. 12, pp. 829-831, Dec. 2005. DOI: 10.1109/ LMWC.2005.860027.

12. Chen, J.; Hong, W.; Chen, X.; Yan, P.; Lai, Q.; $\mathrm{Wu}, \mathrm{K}$. "An LTCC X-band receiver front-end using embedded multilayer substrate integrated waveguide filter," Microwave Opt. Technol. Lett., Vol. 50, No. 2, pp. 285-287, 2008. DOI: 10.1002/mop.23057.

13. Eom, D.-S.; Byun, J.; Lee, H.-Y. "Multilayer substrate integrated waveguide four-way out-of-phase power divider," IEEE Trans. Microwave Theory Tech., Vol. 57, No. 12, pp. 3469-3476, Dec. 2009. DOI: 10.1109/TMTT.2009.2034311.

14. Hong, W.; Liu, B.; Wang, Y.-Q.; Lai, Q.-H.; Tang, Hongjun; Yin, Xiao Xin; Dong, Yuan Dan; Zhang, Yan; Wu, K. "Half mode substrate integrated waveguide: a new guided wave structure for microwave and millimeter wave application," Proc. of Joint 31st Int. Conf. on Infrared Millimeter Waves and 14th Int. Conf. on Terahertz Electronics, 18-22 Sept. 2006, Shanghai, China, IEEE, 2006, p. 219. DOI: 10.1109/ICIMW.2006. 368427.

15. Ali, A. A. M.; Fonseca, N. J. G.; Coccetti, F.; Aubert, H. "Design and implementation of two-layer compact wideband Butler matrices in SIW technology for Ku-band applications," IEEE Trans. Antennas Propag., Vol. 59, No. 2, pp. 503-512, Feb. 2010. DOI: 10.1109/ TAP.2010.2093499.

16. Lee, J.-H.; Pinel, S.; Papapolymerou, J.; Laskar, J.; Tentzeris, M. M. "Low-loss LTCC cavity filters using system-on-package technology at $60 \mathrm{GHz}$," IEEE Trans. Microwave Theory Tech., Vol. 53, No. 12, pp. 3817-3824, Dec. 2005. DOI: 10.1109/TMTT.2005.859864. 
17. Hoer, C. A. "The six-port coupler: A new approach to measuring voltage, current, power, impedance, and phase," IEEE Trans. Instrum., Meas., Vol. 21, No. 4, p. 466-470, 1972. DOI: 10.1109/TIM.1972.4314068.

18. Moldovan, Emilia; Tatu, Serioja-Ovidiu; Gaman, Tamara; Wu, Ke; Bosisio, R. G. "A new 94-GHz six-port collision-avoidance radar sensor," IEEE Trans. Microwave Theory Tech., Vol. 52, No. 3, p. 751-759, Mar. 2004. DOI: $10.1109 /$ TMTT.2004.823533.

19. Tatu, Serioja Ovidiu; Moldovan, Emilia; Wu, Ke; Bosisio, Renato G. "A new direct millimeter-wave six-port receiver," IEEE Trans. Microwave Theory Tech., Vol. 49, No. 12, p. 2517-2522, Dec. 2001. DOI: $10.1109 /$ 22.971644 .

20. Xu, X. Y. "Software defined radio receiver platform based on six-port technology," Doctoral Disser. Ecole Polytechnique Montreal, Canada, 2006. ISBN: 978-0-494-20839-7.

21. Xu, X.; Bosisio, R. G.; Wu, K. “A new six-port junction based on substrate integrated waveguide technology," IEEE Trans. Microwave Theory Tech., Vol. 53, No. 3, pp. 2267-2272, Jul. 2005. DOI: 10.1109/TMTT. 2005.850455 .

22. Liu, B.; Hong, W.; Wang, Y.-Q.; Lai, Q.-H.; Wu, K. "Half mode substrate integrated waveguide (HMSIW) 3-dB coupler," IEEE Microwave Wireless Components Lett., Vol. 17, No. 1, pp. 22-24, Jan. 2007. DOI: 10.1109/ LMWC.2006.887244.

23. Li-nan, W.; Xu-chun, Z.; Chuang-ming, T.; Ming, Z. "A new substrate integrated waveguide six-port circuit," Proc. of Int. Conf. on Microwave and Millimeter Wave Technology, ICMMT, 8-11 May 2010, Chengdu, China. IEEE, 2010, p. 59-61. DOI: 10.1109/ICMMT.20 10.5525288 .

$24 . \quad$ "Microwave Encyclopedia", http://www.microwaves101.com/encyclopedia/siw.cfm, http://www.microwaves101.com/encyclopedia/waveguidemath.cfm)

25. Germain, S.; Deslandes, D.; Wu, K. "Development of substrate integrated waveguide power dividers," Proc. of Canadian Conf. on Electrical and Computer Engineering, 4-7 May 2003, Montreal, QC, Canada. IEEE, 2003, vol. 3, pp. 1921-1924. DOI: 10.1109/CCECE.2003.1226289.

Поступила в редакцию 24.11.2016
26. Levy, R. "Improved single and multiaperture waveguide coupling theory, including explanation of mutual interactions," IEEE Trans. Microwave Theory Tech., Vol. 28, No. 4, pp. 331-338, Apr. 1980. DOI: 10.1109/TMTT.1980.1130073.

27. Cassivi, Y.; Deslandes, D.; Wu, K. "Substrate integrated waveguide directional couplers," Proc. of Asia-Pacific Microwave Conf., Kyoto, Japan, 19-22 Nov. 2002.

28. Bouazza, T. H. C.; Nouri, K.; Bouazza, B. S.; Damou, M.; Becharef, K. "Multilayer substrate integrated waveguide directional coupler," Int. J. Microwave Optical Technol., Vol. 11, No. 4, p. 245-250, July 2016. URI: http://www.ijmot.com/VOL11NO4.ASPX.

29. Kramer, O.; Djerafi, T.; Wu, K. "Dual-layered substrate-integrated waveguide six-port with wideband double-stub phase shifter," IET Microwaves Antennas Propag., Vol. 6, No. 15, pp. 1704-1709, 2012. DOI: 10.1049/iet-map.2012.0272.

30. Doghri, A.; Djerafi, T.; Ghiotto, A.; Wu, K. "Broadband substrate-integrated-waveguide six-port applied to the development of polarimetric imaging radiometer," Proc. of 41st European Microwave Conf., EuMC, 10-13 Oct. 2011, Manchester, UK. IEEE, 2011. DOI: 10.23919/EuMC.2011.6101963.

31. Ding, Y.; Wu, K. "Half-mode substrate integrated waveguide six-port front-end circuits for direct-conversion transceiver design," IEEE MTT-S Int. Microwave Symp. Dig., 1502- June 2008, Atlanta, GA, USA. IEEE, 2008. DOI: 10.1109/MWSYM.2008.4633 267.

32. Chen, J.; Hong, W.; Yan, P.; Liu, B.; Wang, Y.; Wu, K. "Design of a six-port junction using half-mode substrate integrated waveguide," Proc. of Asia-Pacific Microwave Conf., 11-14 Dec. 2007, Bangkok, Thailand. IEEE, 2007. DOI: 10.1109/APMC.2007.4554870.

33. Abdulhadi, A. E.; Suntives, A.; Abhari, R. "Design of a SIW-based data communication system using a SIW six-port receiver," Proc. of IEEE 18th Conf. on Electrical Performance of Electronic Packaging and Systems, 19-21 Oct. 2009, Portland, OR, USA. IEEE, 2009. DOI: 10.1109/EPEPS.2009.5338454. 